PROCEEDINGS OF THE

AMERICAN MATHEMATICAL SOCIETY

Volume 128 , Number 10, Pages 3137-3143

S 0002-9939(00)05468-X

Article electronically published on April 28, 2000

\title{
THE SECTIONAL CATEGORY OF SPHERICAL FIBRATIONS
}

\author{
DON STANLEY \\ (Communicated by Ralph L. Cohen) \\ This paper is dedicated to my son Russell
}

\begin{abstract}
We give homological conditions which determine sectional category, secat, for rational spherical fibrations. In the odd dimensional case the secat is the least power of the Euler class which is trivial. In the even dimensional case secat is one when a certain homology class in twice the dimension of the sphere is -1 times a square. Otherwise secat is two. We apply our results to construct a fibration $p$ such that $\operatorname{secat}(p)=2$ and $\operatorname{genus}(p)=\infty$. We also observe that secat, unlike cat, can decrease in a field extension of $\mathbb{Q}$.
\end{abstract}

\section{INTRODUCTION}

The study of sectional category, or secat, goes back at least to Krasnosel'skii [8] and Yang 14 who studied genus and B-index respectively. These can be considered as special cases of secat. Still the main reference for secat is the paper of Švarc [11] (who also used the term genus). Let $p: E \longrightarrow B$ be a fibration. Then $\operatorname{secat}(p)$ is the least number of open subsets of $B$ over which $p$ has a section that it takes to cover $B$. If $E \simeq *$, then $\operatorname{secat}(p)=\operatorname{cat}(B)+1$ so we see that secat is a generalization of LS category. secat also has many other applications which include critical point theory and embedding theory (see [11]).

This paper is concerned with the secat of fibrations with fibre a sphere. The secat of such fibrations has been previously studied [11. By restricting ourselves to the rational case we can completely solve the problem of determining secat.

We work in the category of spaces having the homotopy type of a CW-complex 9]. For any map $p$ we will always let $\operatorname{Fib}(p)$ denote the homotopy fibre of $p$. We give another definition of secat. They were shown to be equivalent by Švarc [11.

Definition 1.1. Let $p(n): *_{B}^{n} E \rightarrow B$ be the $n$-fold fibrewise join of $p$ with itself (see [7]). If $B$ is a point, we denote $*_{*}^{n} E$ by $*^{n} E$. Then $\sec (p) \leq n$ if and only if $p(n)$ has a section.

The fact that this definition is equivalent to the open set one follows from [7] together with two facts. The fact that $\mathrm{CW}$-complexes have the homotopy type of paracompact spaces and the fact that secat of a fibration induced by a homotopy

Received by the editors December 10, 1998.

1991 Mathematics Subject Classification. Primary 55R25, 55P62; Secondary 55M30.

Key words and phrases. Sectional category, spherical fibrations, rational homotopy theory, Lusternik-Schnirelmann category.

This work was supported by DFG grant Sche 328/2-1. 
equivalence into the base is the same as secat of the original fibration. Observe that our definition of $*^{n} E$ coincides with the usual one. We state a few facts about the $n$-fold fibrewise join.

Proposition 1.2. 1) $p(n)$ is natural in $p$. In other words a diagram

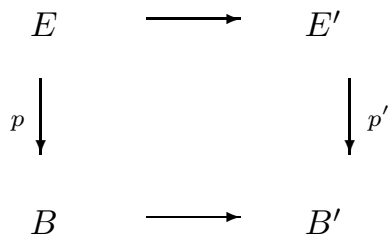

gives us a diagram

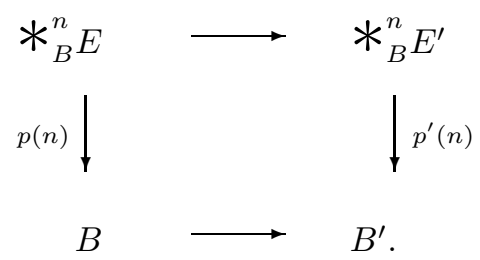

If the first diagram is a pullback, then so is the second.

2) $\operatorname{Fib} p(n) \simeq \mathbb{*}^{n} \operatorname{Fib} p$

Proof. 1) is proved by Doeraene [2] 2) follows from 1) when we let $E \simeq$ Fib $p$ and $B \simeq *$.

We will use the following fact about secat. It is due to Švarc.

Theorem 1.3. Let $p: E \longrightarrow B$ be a fibration. Assume $\left(\operatorname{ker} H^{*}(p)\right)^{r-1} \neq 0$. Then secat $p \geq r$.

Proof. See [1] or [7.

We also need to use the Lusternik-Schnirelmann category of a map.

Definition 1.4. Let $f: X \longrightarrow Y$ be a map. Then

$$
\operatorname{cat}(f)=\min \mid\left\{U_{i} \subset X \mid U_{i} \text { open, } \bigcup U_{i}=X, f \mid u_{i} \simeq *\right\} \mid-1 .
$$

We define $\operatorname{cat}(X)=\operatorname{cat}\left(\mathrm{id}_{X}\right)$.

The following facts about cat $(f)$ are easy to prove.

Proposition 1.5. Let $X, Y, Z$ be spaces and $f^{\prime}, f: X \longrightarrow Y, g: Y \longrightarrow Z$ be maps. Then $\operatorname{cat}(g f) \leq \operatorname{cat}(Y)$. If there exists a cell decomposition of $X$ with $r$ cells, then $\operatorname{cat}(X) \leq r-1$. If $f \simeq f^{\prime}$, then $\operatorname{cat}(f)=\operatorname{cat}\left(f^{\prime}\right)$.

Proof. See [7].

\section{OdD Dimensional Fibre}

Let $F \stackrel{i}{\longrightarrow} E \stackrel{p}{\longrightarrow} B$ be a fibration sequence such that $E$ and $B$ are simply connected and of finite type. Assume $F \simeq S^{2 n+1}$. We work over the rationals. This means that all spaces and cohomology are rational. In this section we show that $\operatorname{secat}(p)$ is the smallest $r$ such that $\operatorname{ker}\left(H^{*}(p)\right)^{r}=0$ (Corollary 2.4). 
Notice that $F$ is a $K(\mathbb{Q}, 2 n+1)$. So it follows from the theory of relative Postnikov systems [13] that $p$ is a principle fibration. (This can also be seen by looking at Sullivan models.) So $p$ is the inclusion of the fibre of a fibration

$$
f: B \longrightarrow K(\mathbb{Q}, 2 n+2) .
$$

In this situation we have the following theorem of Schwartz.

Theorem 2.1. $\operatorname{secat}(p)=\operatorname{cat}(f)+1$.

Proof. See 11], Theorem 19" or [7].

Definition 2.2. $f: B \longrightarrow K(\mathbb{Q}, 2 n+2)$ corresponds to $\alpha \in H^{2 n+2}(B)$. We call $\alpha$ the Euler class of $p$. Since $B$ is simply connected, this is the same as the usual Euler class.

Theorem 2.3. Let $\alpha$ be the Euler class of $p$. Let $r$ be the least integer such that $\alpha^{r}=0$. Then $\operatorname{secat}(p)=r$.

Proof. Assume $\alpha^{r}=0$. Then $f$ factors through the fibre $G$ of the map

$$
K(\mathbb{Q}, 2 n+2) \longrightarrow K(\mathbb{Q},(2 n+2) r)
$$

which represents $\iota^{r} \in H^{(2 n+2) r}(K(\mathbb{Q}, 2 n+2))$. Of course $G$ is just the $(2 n+2)(r-1)$ skeleton of $K(\mathbb{Q}, 2 n+2)$ and so cat $(G) \leq r-1$. (To see cat $(G) \leq r-1$ calculate that $H^{*} G \cong P(a) /\left(a^{r}\right)$. So $G$ can be built with $r$ cells and so $\operatorname{cat}(G) \leq r-1$ by Proposition 1.5.) Therefore by Proposition $1.5 \operatorname{cat}(f) \leq \operatorname{cat}(G) \leq r-1$. So $\operatorname{secat}(p) \leq r$. But $\alpha^{r-1} \neq 0$ so by Theorem 1.3 secat $(p) \geq r$. We conclude that $\operatorname{secat}(p)=r$.

Another way to phrase the result of the theorem is:

Corollary 2.4. $\operatorname{secat}(p)$ is the smallest $r$ such that $\left(\operatorname{ker} H^{*}(p)\right)^{r}=0$.

Proof. Assume $\left(\operatorname{ker} H^{*}(p)\right)^{r}=0$ but $\left(\operatorname{ker} H^{*}(p)\right)^{r-1} \neq 0$. Then by Theorem 1.3 $\operatorname{secat}(p) \geq r$. But the $r$-th power of the Euler class is trivial so secat $(p) \leq r$.

\section{EVEN DIMENSIONAL FIBRE}

Again let $F \stackrel{i}{\longrightarrow} E \stackrel{p}{\longrightarrow} B$ be a fibration sequence such that $E$ and $B$ are simply connected and of finite type. Assume $F \simeq S^{2 n}$. Again we work over the rationals. This time we need to make use of Sullivan models. For information on Sullivan models see [5], [12] or [10]. $(\Lambda V, d)$ denotes the free commutative differential graded algebra on a graded vector space $V$ with differential $d$. For a graded set $(a(1), \ldots, a(n)),(\Lambda(a(1), \ldots, a(n)), d)$ denotes $(\Lambda V, d)$ where $V$ is the vector space with basis $(a(1), \ldots, a(n))$. For vector spaces $V$ and $W,(\Lambda V \otimes \Lambda W, d)$ is the same as $(\Lambda(V \oplus W), d)$ with the added assumption that $d(V) \subset \Lambda V$. For convenience we will use the same notation for a map and a model of the map.

Lemma 3.1. There exists a model for $E$ of the form

$$
(\Lambda V \otimes \Lambda(a, b), d)
$$

such that $d a=0$ and $d b=a^{2}+\alpha$ for some cycle $\alpha \in \Lambda V$. Also the inclusion

$$
p:(\Lambda V, d) \longrightarrow(\Lambda V \otimes \Lambda(a, b), d)
$$

models the map $p$. Given the form of the differential and the fact that the inclusion models $p$ the homology class of the cycle $\alpha$ is determined. 
Proof. $(\Lambda(a, b), d)$ with $d b=a^{2}$ and $d a=0$ is a model of $F$. Therefore there exists a model $(\Lambda V \otimes \Lambda(a, b), d)$ for $E$. First we show that we can assume $d a=0$. There exist $\delta, \gamma \in \Lambda V$ such that

$$
d b=a^{2}+\gamma a+\delta
$$

So

$$
0=d d b=2 a d a+a d \gamma+\text { terms without } a .
$$

Therefore $d a=-\frac{1}{2} d \gamma$. So by changing basis in a way compatible with $p$ we can assume $d a=0$. In the new basis there still exist $\delta, \gamma$ so that (1) holds. Now (2) implies $d \gamma=0$. So again we can change basis compatibly with $p$ so that $d b=a^{2}+\delta$ for some $\delta \in \Lambda V$.

Assume that there exists another model $\left(\Lambda V \otimes \Lambda\left(a^{\prime}, b^{\prime}\right), d^{\prime}\right)$ for $E$ compatible with the inclusion and such that $d^{\prime} a^{\prime}=0$ and $d^{\prime} b^{\prime}=a^{\prime}+\alpha^{\prime}$ for some cycle $\alpha^{\prime} \in \Lambda V$. So then there is an equivalence

$$
f:(\Lambda V \otimes \Lambda(a, b), d) \longrightarrow\left(\Lambda V \otimes \Lambda\left(a^{\prime}, b^{\prime}\right), d^{\prime}\right)
$$

that is compatible with the inclusion of $\Lambda V$. So by multiplying $a^{\prime}$ by -1 if necessary we get that $f(a)=a^{\prime}+\gamma$ for some cycle $\gamma \in \Lambda V$. But since $f(d b)=f\left(a^{2}\right)=$ $\left(a^{\prime}\right)^{2}+2 a \gamma+\gamma^{2}$ is a boundary it follows that $\gamma$ must be a boundary and so we can change $f$ and assume that $\gamma=0$. Again using the fact that $f(d b)$ is a boundary we see that $d b \simeq d b^{\prime}$ in $\Lambda V \otimes \Lambda(a)$. Therefore $\alpha \simeq \alpha^{\prime}$.

Lemma 3.2. $\operatorname{secat}(p) \leq 2$.

Proof. Represent $p$ as in Lemma 3.1. Then we have a commutative diagram

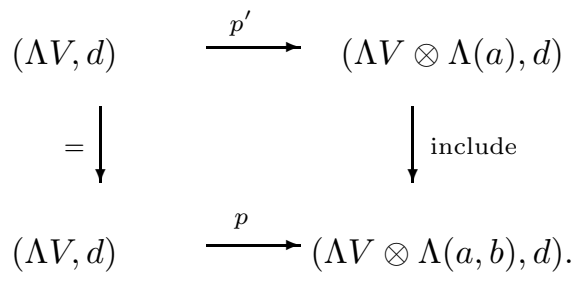

Since $d a=0$, secat $\left(p^{\prime}\right)=1$. Taking fibrewise joins we get a commutative diagram

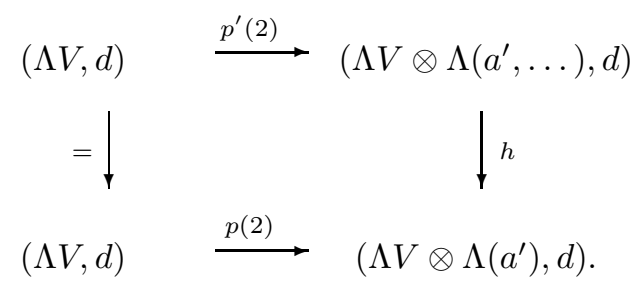

The dimension of $a^{\prime}$ is $4 n+1$ and all other generators of the model of $\operatorname{Fib}\left(p^{\prime}(2)\right)$ are in higher dimensions. Remember that Fib $p \simeq S^{2 n}$. Therefore Fib $p(2) \simeq S^{4 n+1}$. Also the induced map $\operatorname{Fib}\left(p^{\prime}\right) \longrightarrow \operatorname{Fib}(p)$ is an $H_{2 n}$ isomorphism. It follows that we can represent the fibrations and maps so that $h\left(a^{\prime}\right)=a^{\prime}$. Since secat $\left(p^{\prime}\right)=$ $1<2$ there exists a map $r^{\prime}$ such that $r^{\prime} p^{\prime}(2)=\mathrm{id}$. Therefore, by changing basis if necessary, we may assume that $d a^{\prime}=0$. So there is an $r:\left(\Lambda V \otimes \Lambda\left(a^{\prime}\right), d\right) \longrightarrow(\Lambda V, d)$ such that $r p(2)=$ id. Thus $\operatorname{secat}(p) \leq 2$. 
Theorem 3.3. Let $p: E \longrightarrow B$ be a fibration with fibre $S^{2 n}$. Let $p:(\Lambda V, d) \longrightarrow$ $(\Lambda V \otimes \Lambda(a, b), d)$ be a model of $p$ such that $d a=0$ and $d b=a^{2}+\alpha$, where $\alpha$ is a cycle in $\Lambda V$. Then secat $(p)=1$ if $\alpha$ represents $-r^{2}$ for some $r \in H^{*} B$. Otherwise $\operatorname{secat}(p)=2$.

Proof. The statement that $p$ can be modeled in the way stated in the theorem is just Lemma 3.1. Also recall that the homology class of $\alpha$ is determined. Let $r \in H^{*} B$ and assume that $\alpha$ represents $-r^{2}$. Let $\beta \in \Lambda V$ represent $r$. Then there exists $\gamma \in \Lambda V$ such that $d \gamma=\beta^{2}+\alpha$. We define $r:(\Lambda V \otimes \Lambda(a, b), d) \longrightarrow(\Lambda V, d)$ by

$$
\begin{aligned}
\left.r\right|_{\Lambda V} & =\mathrm{id} \\
r(a) & =\beta \\
r(b) & =\gamma .
\end{aligned}
$$

Clearly $r p=$ id. So $\operatorname{secat}(p)=1$.

Now assume $\operatorname{secat}(p)=1$ Then there is a map

$$
r:(\Lambda V \otimes \Lambda(a, b), d) \longrightarrow(\Lambda V, d)
$$

such that $r p=\mathrm{id}$.

Let $r(a)=\beta$ and $r(b)=\gamma$. Notice $d \beta=0$. We also have the equation in $\Lambda V$, $\beta^{2}+\alpha=r d b=d r b=d \gamma$. Therefore $\beta^{2}+\alpha \simeq 0$ and so $-\beta^{2} \simeq \alpha$. In other words $\alpha$ represents $-r^{2}$ for $[\beta]=r \in H^{*} B$. The last statement of the theorem then follows easily from Lemma 3.2.

Remark 1. As pointed out by the referee the fact that we can assume that $d a=0$ in Lemma 3.1 is just the fact that the Euler class of a spherical fibration with an even dimensional fibre is torsion (in fact 2-torsion). Furthermore part of the content of Theorem 3.3 is to pin down the secondary (and only other) rational obstruction to such a fibration having a section.

Example. Consider the map

$$
p:(\Lambda(c), d) \longrightarrow(\Lambda(c, a, b), d)
$$

where $d a=0$ and $d b=a^{2}+\alpha c^{2}$. Then secat $(p)=1$ if and only if $\alpha=-k^{2}$ for some $k \in \mathbb{Q}$. This example makes it clear that $\operatorname{secat}(p)$ can decrease in a field extension. This is in contrast to the situation for LS category where Hess [6], Theorem 4 showed that cat is independent of field extension of $\mathbb{Q}$.

\section{An application to genus}

In this section we apply the result of the last section together with a result of Gatsinzi [4] to construct maps $f$ with $\operatorname{secat}(f)=2$ and genus $(f)=\infty$. We first define genus and give a characterization of it in terms of the cat of the classifying map of the fibration. Let $p: E \longrightarrow B$ be a fibration.

Definition 4.1. An open cover $\left\{U_{i}\right\}$ of $B$ such that, for every $i,\left.p\right|_{U_{i}}$ is equivalent to a product fibration is called $p$ trivial.

$$
\operatorname{genus}(p)=\min _{\left\{U_{i}\right\} \text { trivial }}\left|\left\{U_{i}\right\}\right| .
$$

It is clear from the definition that $\operatorname{secat}(p) \leq \operatorname{genus}(p)$.

We can give another characterization of genus which generalizes [11], Theorem 19 " to non-principal fibrations. The method of proof is the same. 
Theorem 4.2. Let $F$ denote the fibre of $p$ and $\phi: B \longrightarrow B$ aut $F$ its classifying map. Then $\operatorname{genus}(p)=\operatorname{cat}(\phi)+1$.

Proof. It follows from [1] that $\left.\phi\right|_{U_{i}}: U_{i} \longrightarrow B$ aut $F$ is inessential if and only if $\left.p\right|_{U_{i}}$ is equivalent to a product fibration. The result then follows easily.

For the rest of this section we work over the rationals.

Theorem 4.3 (4]). There are maps

$$
f: K(Q, 4 n) \longrightarrow B \text { aut } S^{2 n}
$$

that are non-trivial on $\pi_{*}$.

Proof. This is a special case of a theorem in 4]. There it is shown that there is such a map for any Gottlieb element of any coformal space.

Corollary 4.4. Any fibration $p$ corresponding to a nontrivial $f: K(Q, 4 n) \longrightarrow$ $B$ aut $S^{2 n}$ has genus $(p)=\infty$.

Proof. The mapping theorem [3] implies that cat $(f)=\infty$. (non-triviality implies injectivity in this case.) Theorem 4.2 then implies genus $(p)=\infty$.

Theorem 4.5. The fibrations $p$ corresponding to non-trivial maps $f$ of Theorem 4.3 are represented by $K S$ extensions of the form

$$
\Lambda(c) \longrightarrow \Lambda(a, b, c)
$$

where $d a=d c=0, d b=a^{2}+\alpha c$ and $\alpha \in \mathbb{Q}^{\times}$. So $\operatorname{secat}(p)=2$ and $\operatorname{genus}(p)=\infty$.

Proof. From Lemma 3.1 and for dimension reasons the fibration must be representable in the form stated in the theorem for some $\alpha \in \mathbb{Q} . \alpha \neq 0$ since otherwise the fibration would be trivial. That $\operatorname{secat}(p)=2$ follows directly from Theorem 3.3. That $\operatorname{genus}(p)=\infty$ is just Corollary 4.4.

Remark 2. For every $r>1$, consider the fibration $p$ represented by the KS extension

$$
\Lambda(c, e) \longrightarrow \Lambda(c, e, a, b)
$$

where $d e=c^{r}, d c=d a=0$ and $d b=a^{2}+\alpha c, \alpha \in \mathbb{Q}^{\times}$. It is not hard to see that $\operatorname{secat}(p)=2, \operatorname{genus}(p)=r$.

\section{REFERENCES}

[1] M.G. Barratt, V.K.A.M. Gugenheim and J.C. Moore, On semi-simplicial fibre bundles, Amer. Jour. Math. 81 (1959), 629-657. MR 22:1895

[2] J.-P. Doeraene, L.S.-category in a model category, Jour. Pure and App. Alg. 84 (1993), 215-261. MR 94b:55017

[3] Y. Felix and S. Halperin, Rational L.S. category and its applications, Trans. Amer. Math. Soc. 273 (1982), 1-37. MR 84h:55011

[4] J.-B. Gatsinzi, LS-category of classifying spaces II, Bull. Belg. Math. Soc. 3 (1996), 243-248. MR 97d:55022

[5] S. Halperin, Lectures on minimal models, Memoire de la Société Math. de France (N. S.), 9/ 10 (1983). MR 85i:55009

[6] K. Hess, A proof of Ganea's conjecture for rational spaces, Topology 30 (1991), 205-214. MR 92d:55012

[7] I. M. James, On category in the sense of Lusternik-Schnirelmann, Topology, 17 (1978), 331-348. MR 80i:55001

[8] M. A. Krasnolsel'skii, Topological methods in the theory of non-linear integral equations, GITTL, Moscow (1956), (Russian). MR 28:2414 
[9] J. Milnor, On spaces having the homotopy type of a CW-complex, Trans. Amer. Math. Soc. 90 (1959), 272-280. MR 20:6700

[10] D. Sullivan, Infinitesimal computations in topology, Publications Mathématiques de l'Institute des Hauts Etudes Scientifiques, 47 (1977), 269-331. MR 58:31119

[11] A. S. Švarc, The genus of a fiber space, Am. Math. Soc. Transl. 55 (1966), 49-140. MR 21:1598

[12] D. Tanré, Homotopie Rationnelle: Modéles de Chen, Quillen, Sullivan. Lecture Notes in Math. 1025, (1983), Springer-Verlag. MR 86b:55010

[13] G.W. Whitehead, Elements of Homotopy Theory, Springer-Verlag (1978). MR 80b:55001

[14] C.-T. Yang, On theorems of Borsuk-Ulam, Kakutani-Yamabe-Yujobo and Dupon I and II, Ann. of Math. 60 (1954), 262-282, and 62 (1955), 271-283. MR 16:502d MR 17:289e

II Mathematisches Institut, Freie Univerität Berlin, Arnimallee 3, D-14195 Berlin, GERMANY

E-mail address: stanley@math.fu-berlin.de

Current address: Max-Plank-Institut für Mathematik, Vivatsgasse 7, D-53111 Bonn, Germany

E-mail address: stanley@mpim-bonn.mpg.de 